\title{
AN ASSESSMENT OF SEMANTIC INFORMATION AUTOMATICALLY EXTRACTED FROM MACHINE READABLE DICTIONARIES
}

\author{
Jean Véronis ${ }^{1.2}$ and Nancy Ide' \\ 1 Department of Computer Science \\ VASSAR COLLEGE \\ Poughkeepsie, New York 12601 (U.S.A.) \\ ${ }^{2}$ Groupe Représentation et Traitement des Connaissances \\ CENTRE NATIONAL DE LA RECHERCHE SCIENTIFIQUE \\ 31, Ch. Joseph Aiguier \\ 13402 Marseille Cedex 09 (France)
}

\begin{abstract}
In this paper we provide a quantitative evaluation of information automatically extracted from machine readable dictionaries. Our results show that for any one dictionary, $55-70 \%$ of the extracted information is garbled in some way. However, we show that these results can be dramatically reduced to about $6 \%$ by combining the information extracted from five dictionaries. It therefore appears that even if individual dictionaries are an unreliable source of semantic information, multiple dictionarics can play an important role in building large lexical-semantic databases.
\end{abstract}

\section{INTRODUCTION}

In recent ycars, it has become increasingly clear that the limited size of existing computational lexicons and the poverty of the semantic information they contain represents one of the primary botllenecks in the development of realistic natural language processing (NLP) systems. The need for extensive lexical and scmantic databases is evident in the recent initiation of a number of projects to construct massive generic lcxicons for NLP (project GENELEX in Europe or EDR in Japan).

The manual construction of large lexical-scmantic databases demands cnormous human resources, and there is a growing body of rescarch into the possibility of automatically extracting at least a part of the required lexical and semantic information from cveryday dictionarics. Everyday dictionaries are obviously not structured in a way that cnables their immediate use in NLP systems, but several studies have shown that relatively simple procedures can be used to extract laxonomics and various other semantic rclations (for cxample, Amsler, 1980; Calzolari, 1984; Chodorow, Byrd, and Heidom, 1985; Markowitz, Ahlswede, and Evens, 1986; Byrd et al., 1987; Nakamura and Nagao, 1988; Veronis and Ide, 1990; Klavans, Chodorow, and Wacholder, 1990; Wilks el al., 1990).

However, it remains to be seen whether information automatically extracted from dictionaries is sufficiently complete and coherent to be actually usable in NLP systems. Although there is concern over the quality of automatically extracted lexical information, very few cmpirical studies have attempted to assess it systematically, and those that have done so have been restricted to consideration of the quality of grammatical information (c.g., Akkerman, Maserecuw, and Meijs, 1985). No cvaluation of automatically extracted semantic information has been published.

The authors would like to thank Lisa Lassck and Anne Gilman for their contribution to this work.
In this paper, we report the results of a quantitative evaluation of automatically extracted semantic data. Our results show that for any one dictionary, 55-70\% of the extracted information is garbled in some way. These results at first call into doubt the validity of automatic extraction from dictionaries. However, in section 4 we show that these results can be dramatically reduced to about $6 \%$ by several means--most significantly, by combining the information extracted from five dictionaries. It therefore appears that even if individual dictionaries are an unreliable source of semantic information, multiple dictionaries can play an important role in building large lexical-scmantic databases.

\section{METHODOLOGY}

Our strategy involves automatically extracting hypernyms from five English dictionaries for a limited corpus. To determine where problems exist, the resulting hicrarchies for cach dictionary are compared to an "ideal" hierarchy constructed by hand. The five dictionarics compared wcre: the Collins English Dictionary (CED), the Oxford Advanced Learner's Dictionary (OALD), the COBUILD Dictionary, the Longman's Dictionary of Contemporary English (LDOCE) and the Webster's 9/h Dictionary (W9).

We begin with the most straightforward case in order to detcrmine an upper bound for the results. We deal with words within a domain which poses few modelling problems, and we focus on hyperonymy, which is probably the least arguable scmantic relation and has been shown to be the easiest to extract. If the results are poor under such favorable constraints, we can foresee that they will be poorer for more complex (abstract) domains and less clcarly cut relations.

An idcal hierarchy probably does not exist for the entire dictionary; however, a fair degree of consensus seems possible for carefully chosen terms within a very restricted domain. We have therefore selected a corpus of one hundred kitchen utensil terms, each representing a concrcte, individual objecl-for example, cup, fork, saucepan, decanter, ctc. All of the terms are count nouns. Mass nouns, which can cause problems, have becn excluded (for example, the mass noun cutlery is not a hypernym of $k n i f e$ ). Other idiosyncratic cases, such as chopsticks (where it is not clear if the utensil is one object or a pair of objects) have also been climinated from the corpus. This makes it easy to apply simple tests for hyperonymy, which, for instance, cnable us to say that $Y$ is a hypermym of $X$ if "this is an $X$ " entails but is not entailed by "this is a Y" (Lyons, 1963).

Chodorow, Byrd, and Heidorn (1985) proposed a heuristic for extracting hypernyms which exploits the fact that definitions for nouns typically give a hypernym 
term as the head of the defining noun phrase. Consider the following examples:

$\begin{array}{ll}\text { dipper } & \text { a ladle used for dipping... [CED] } \\ \text { ladie } & \text { a long-handled spoon... [CED] } \\ \text { spoon } & \text { a metal, wooden, or plastic utensil... [CED] }\end{array}$

In very general terms, the heuristic consists of extracting the word which precedes the first preposition, relative pronoun, or participle encountered in the definition text. When this word is "empty" (c.g. one, any, kind, class) the true hypernym is the head of the noun phrase following the preposition of:

slice any of various wensils... [CED]

Automatically extracted hicrarchics are necessarily langled (Amsler, 1980) because many words are polyscmous. For example, in the CED, the word pan has the following senses (among others):

$\begin{array}{ll}\operatorname{pan}^{1} & 1 . \text { a wide metal vessel ... [CED] } \\ \operatorname{pan}^{2} & 1 \text { the leaf of the betcl tree... [CED] }\end{array}$

The CED also gives pan as the hypernym for saucepan, which taken together yiclds the hicrarchy in figure 1.a The tangled hicrarchy is problematic becausc, following the path upwards from saucepan, we find that saucepan can be a kind of leaf. This is clearly erroneous. A hicrarchy utilizing senses rather than words would not be tangled, as shown in figure 1.b.

In our study, the hicrarchy was disambiguated by hand. Sense disambiguation in dictionary definitions is a difficult problem, and we will not address it here; this problem is the focus of much current rescarch and is considered in depth clsewhere (c.g., Byrd et al., 1987; Byrd, 1989; Véronis and Ide, 1990; Klavans, Chodorow, and Wacholder, 1990; Wilks et al., 1990).

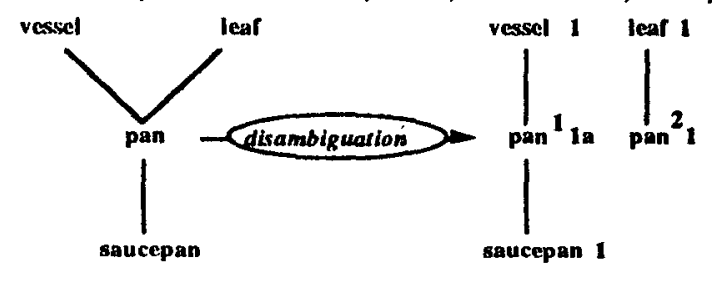

a) word hierarchy

b) sense hlerarchy

Figure 1 : Sense-tangled hierarchy

\section{EVALUATION}

Hierarchies constructed with methods such as those outlined in section 2 show, upon close inspection, scveral serious problems. In this section, we describe the most pervasive problems and give their frequency in our five dictionaries. The problems fall into two general types: those which arise because information in the dictionary is incomplete, and those which are the result of a lack of distinction among terms and the lack of a one-to-one mapping between terms and concepts, especially at the highest levels of the hicrarchy.

\subsection{Incomplete information}

The information in dictionaries is incomplete for two main reasons. First, since a dictionary is typically the product of several lexicographers efforts and is constructed, revised, and updated over many ycars, there exist inconsistencies in the criteria by which the hypernyms given in definition texts are chosen. In addition, space and readability restrictions, on the onc hand, and syntactic restrictions on phrasing, on the other, may dictate that certain information is unspecified in definition texts or left to be implied by other parts of the definition.

\subsubsection{Attachment too high : $21-34 \%$}

The most pervasive problem in automatically extracted hierarchies is the attachment of terms too high in the hierarchy. It occurs in $21-34 \%$ of the definitions in our sample from the five dictionaries (figure 8). For example, while pan and bottle are vessels in the CED, cup and bowl are simply containers, the hypernym of vessel. Obviously, "this is a cup" and "this is a bowl" both entail (and are not entailed by) "this is a vessel". Further, other dictionaries give vessel as the hypernym for cup and bowl. Therefore, the attachment of cup and bowl to the higher-level term conlainer scems to be an inconsistency within the $C E D$.

The problem of altachment too high in the hicrarchy occurs relatively randomly within a given dictionary. In dictionaries with a controlled definition vocabulary (such as the $L D O C E$ ), the problem of attachment at high levels of the hicrarchy results also from a lack of terms from which to choose. For example, ladle and dipper are both attached to spoon in the $L D O C E$, although "this is a dipper" cntails and is not entailed by "this is a ladle". There is no way that dipper could be defined as a ladle (as, for instance, in the CED), since ladle is not in the defining vocabulary. As a result, hicrarchies extracted from the $L D O C E$ are consistently flat (figure 7).

\subsubsection{Absent hypernyms : $\mathbf{0 - 3 \%}$}

In some cases, strategies like that of Chodorow, Byrd and Heidorn yicld incorrect hypernyms, as in the following definitions:

$\begin{array}{ll}\text { grill } & \text { A grill is a part of a cooker... [COBUIL.D] } \\ \text { corkscrew } & \text { a pointed spiral piece of metal... [W9] } \\ \text { dinner service a complete set of plates and dishes... [LDOCE, } \\ \text { [LDt included in our corpus] }\end{array}$

The words part, piece, set, are clcarly not hypernyms of the defined concepts: it is virtually meaningless to say that grill is a kind of part, or that corkscrew is a kind of piece. In these cases, the head of the noun phrase serves to mark another relation: part-whole, member-class, etc. It is casy to reject these and similar words (member, series, etc.) as hypernyms, since they form a closed list (Klavans, Chodorow, and Wacholder, 1990). However, excluding these words lcaves us with no hypernym. We call these "absent hypernyms"; they occur in $0-3 \%$ of the definitions in our sample corpus (figure 8).

The absence of a hypernym in a given definition text docs not necessarily imply that no hypernym exists. For example, "this is a corkscrew" clearly entails (and is not entailed by) "this is a device" (the hypernym given by the COBUILD and the $C E D$ ). In many cases, the lack of a hypernym seems to be the result of concem over space and/or readability. We can imagine, for example, that the definition for corkscrew could be more fully specified as "a device consisting of a pointed spiral piece of metal..." In such cases, lexicographers rely on the reader's ability to deduce that something made of metal, with a handle, used for pulling corks, can be called a device. However, for some terms, such as cutlery or dinner service, it is not clear that a hypernym exists. Note that we have voluntarily cxcluded problematic terms of this kind from our corpus, in order to restrict our evaluation to the best casc.

\subsubsection{Missing overlaps : $8-14 \%$}

Another problem results from the necessary choices that lexicographers must make in an attempt to specify a 
single superordinate, when concepts in the real world overlap frecly. For instance, a saucepan can be said to be a pol as well as a pan. "This is a saucepan" entails both "this is a pot" (the hypernym given by the CED and W9) as well as "this is a pan" (the hypernym given by the $\angle D O C E, O A L D$, and COBUILD). On the other hand, "this is a pot" does not entail and is not entailed by "this is a pan", which is to say that pot and pan are not synonyms, nor is one the hypernym of the other. In terms of classes, pan and pot are distinct but overlapping, and saucepan is a subset of their intersection (figure 2.a). This is no longer a strict hicrarchy since it includes merging branches (figure 2.b). We will call it an "overlapping hicrarchy". Although a tree representation of such a hicrarchy is impossible, it presents no problems on either logical or computational grounds.
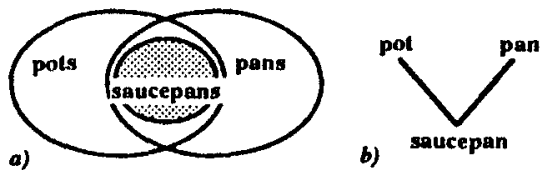

Figure 2. Overlapping hlerarchy

Assuming the above relations, it would be more logically correct to phrase the definition of saucepan as "a pan AND a pol...". However, lexicographers never use "and" in this way, but usually give only one of the alternatives. For example, each of the five dictionaries in our study chooses either pol or pan as the genus term for saucepan. When this occurs, one of the hypernyms is missing. This problem arises in our sample corpus relatively frequently, 8-14\% of the time depending on the dictionary (figure 8).

\subsection{Difficulties at higher levels}

At the higher levels of the hierarchy, terms necessarily become more general, and they often become less clearly defined. For example, most people will agree on whether some object falls into the category fork or spoon, but there is much less agrecment on what objects are implements or ulensils. In addition, at the higher levels some concepts simply lack a term to designate them exactly. As a result; there is confusion at the higher levels of hicrarchics implicit in diclionary definitions.

\subsubsection{OR-conjoined heads : $\mathbf{7 - 1 0} \%$}

For $7-10 \%$ of the terms in our corpus, definitions give a list of head nouns separated by the conjunction or, as in the following:

utensll an implement, tool or container... [CED]

In this case, none of the three alternatives is a hypernym of utensil. First, it is clearly not true that "this is a utensil" entails "this is a container". For the other two, it is not clear whether or not "this is a utensil" entails "this is a tool" and "this is an implement", and it is even less clear that the reverse entailments do not apply. Regarding the three terms as hypernyms of utensil would produce the hierarchy in figure 3. However, by cnumerating the paths upwards from spatula (defined as a utensi), we see that spatula is a kind of conlainer, which is obviously incorrect.

This solution amounts to regarding the class of utensils as the intersection of the classes of implements, tools, and containers. Regarding the conjunction or as denoting the union of these classes would be more correct on logical grounds, since if $X$ is included in $A$ or $X$ is included in $B$, then $X$ is included in $A \cup B$. This relation cannot be fitted into a trec, but it can be pictured as in figure 4. However, this does not help to determine whether spatula is an implement, tool, or container, or some subsct of the threc. In any case, lexicographers do not use or with a consistent, mathematical meaning. Or-conjoincd heads appear nol to be usable in constructing hierarchical trees without considerable manipulation and addition of information.

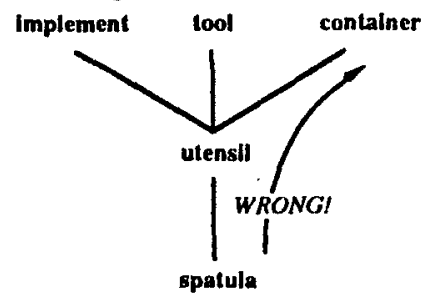

Figure 3 : problematic hierarchy

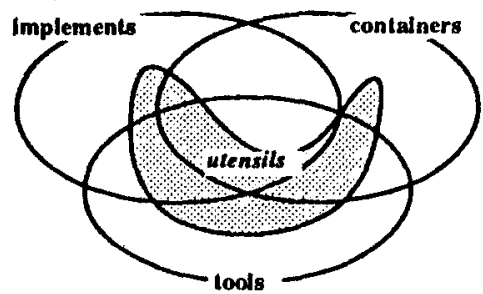

Figure 4. $O R$ as class union

\subsubsection{Circularity : $7-11 \%$}

It is well known that circularity exists in dictionary definitions, especially when concepts are high up in the hicrarchy. For instance, consider the definitions below:

tool Implement a piece of equipment; tool or utensil. [CED] utensll an implement, lool or container... [CED]

Circular definitions yicld hicrarchies containing loops (figure 5.a). Unlike merging branches, loops have no interpretation in terms of classes. A loop asserts both that $A$ is a sub-class of $B$ and $B$ is a sub-class of $A$, which yiclds $A:=B$. This is why Amsler (1980) suggests merging circularly-defined concepts and regarding them as synonyms (figure 5.b).

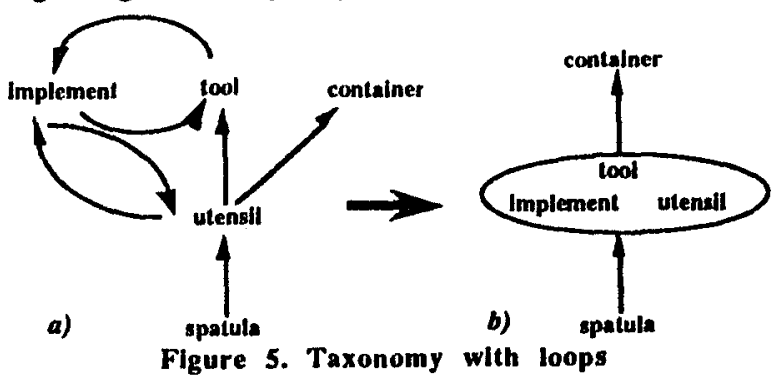

However, in most cases this solution leads to erroneous results; it is clear, for example, that many implements, tools, and utensils (c.g., spatula) are not containers. This problem is similar to the one cited above in section 3.2.1. If dictionary definitions are to be interpreted in terms of set theoretical relations, a more complex mathematical treatment is required. The definitions above can be represented by the following relations:

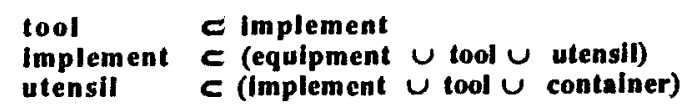
which, once solved, do not equatc tool, implement, and utensil, but instead define the overlapping classes in figure 6. This represcntation is clearly more sound on logical grounds. It still docs not indicate exactly 
where spalula should appear (since we have no indication that it is not a con(ainer), but at least it shows that there may be some utensils which are not containers.

Although this representation is more intuitively accurate than the representation in figure 5.b, ultimately it goes too far in delineating the relations among terms. In actual use, the distinctions among terms are much less clcar-cut than figure 6 implies. For instance, the figure indicates that all tools that are containcrs are also implements, but it is certainly not clear that humans would agrec to this or use the terms in a manner consistent with this specification. Dictionaries themsclves do not agree, and when taken formally they yicld very different diagrams for higher level concepts.

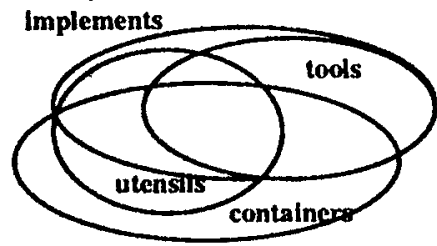

Figure 6. Solving "loops"

Figure 8 shows that $7-11 \%$ of the definitions use a hypemym that is itself defined circularly.

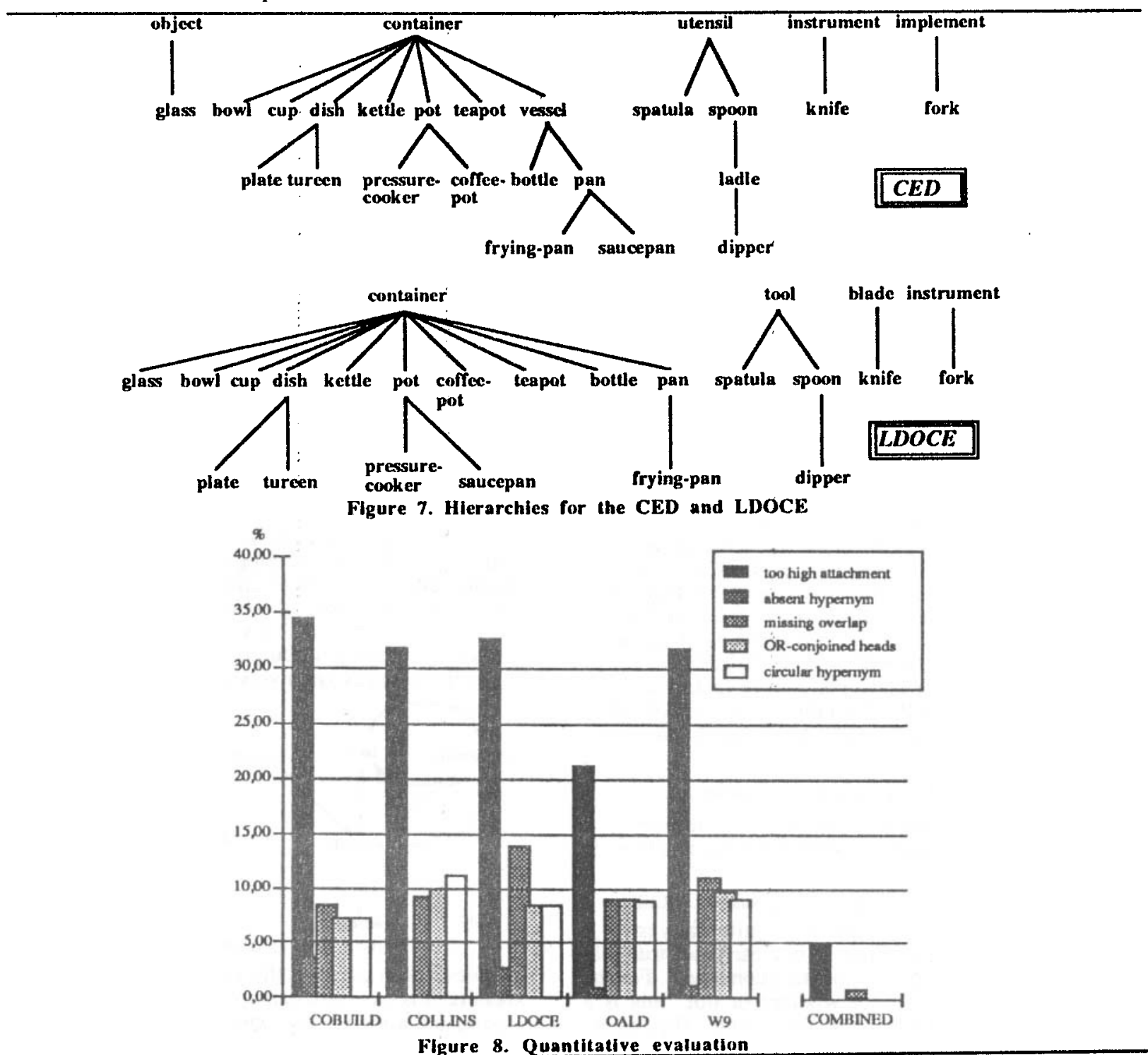

\subsection{Summary}

Altogether, the problems described in the sections above yield a $55-70 \%$ error rate in automatically extracted hierarchies. Given that we have altempted to consider the most favorable case, it appears that any single dictionary, taken in isolation, is a poor source of automatically extracted semantic information. This is made more evident in figure 7 , which demonstrates the marked differences in hierarchies extracted from the $C E D$ and $L D O C E$ for a small subsel of our corpus. A summary of our results appears in figure 8.

\section{REFINING}

We have concluded that hierarchies extracted using strategies such as that of Chodorow, Byrd, and Heidom are seriously flawed, and are therefore likely to be unusable in NLP systems. However, in this section we discuss various means to refine automatically extracted hicrarchies, most of which can be performed automatically. 


\begin{tabular}{|l|c|c|c|c|c|c|}
\hline WOKD & COBUILD & COLLINS & LDOCE & OALD & W9 & Comblned \\
\hline Iadle & spoon & spoon & spoon & spoon & spoon & spoon \\
\hline basin & container & container & container & bowl & vessel & bowl \\
\hline ewer & jug & jug OR pitcher & container & pitcher & pitcher OR jug & pitcher \\
\hline saucepan & pol & pan & pot & pot & pan & pot AND pan \\
\hline grlll & (absent) & device & (absent) & device & utensil & device AND utcnsil \\
\hline fork & tool & implement & instrument & implement & implement & tool, implement AND instrument \\
\hline
\end{tabular}

Figure 9. Merging hierarchles

\subsection{Merging dictionaries}

It is possible to use information provided in the differentiae of definition texts to refine hicrarchies; for cxample, in the definition

vessel any object USISD AS a container... [CED]

the automatically extracted hypernym is object. However, some additional processing of the definition tcxt enables the extraction of container following the phrase "used as". It is also possible to use other definitions. For example, the CED does not specify that knife and spoon are implements, but this information is provided in the definition of cullery:

cutlery implements used for eating SUCH AS knives, forks, and spoons. [CED]

The extraction of information from differentiae demands some extra parsing, which may be difficult for complex definitions. Also, further research is required to detcrmine which phrases function as markers for which kind of information, and to determine how consistent their use is. More importantly, such information is sporadic, and its extraction may require more effort than the results warrant. We therefore seek more "brute force" methods to improve automatically extracted hicrarchics.

Onc of the most promising strategies for refining extracted information is the use of information from several dictionaries. Hierarchics derived from individual dictionaries suffer from incompleteness, but $i t$ is extremely unlikely that the same information is consistently missing from all dictionaries. For instance, the $C E D$ attaches cup to container, which is too high in the hierarchy, while the W9 attaches it lower, to vessel. It is therefore possible to use taxonomic information from several dictionaries to fill in absent hypernyms, missing links, and to reclify cases of too high attachment.

To investigate this possibility, we merged the information extracted from the five English dictionarics in our databasc. The individual data for the five dictionaries was organized in a table, as in figure 9. Merging these hierarchics into a single hierarchy was accomplished automatically by applying a simple algorithm, which scans the table line-by-line, as follows:

1) regard cells containing multiple heads conjoined by or as null, since, as we saw in section 3.2.1, they do not reliably provide a hypernym.

2) if all the cells agree (as for ladle), keep that term as the hypernym. Otherwise:

a) if a term is a hypernym of another term in the linc, ignore it.

b) take the remaining cell or cells as the hypernym(s).

This algorithm must be applied recursively, since, for example, it may not yet be known when evaluating basin that container is a hypernym of vessel, and vessel is a hypemym of bowl, until those terms are themsclves processed. Therefore, several passes through the table are required. Note that if after applying the algorithm several terms are left as hypernyms for a given word, we effectively create an overlap in the hierarchy. For example, saucepen is attached to both pot and pan, and fork is attached to tool, implement, and instrument.

We evaluate the quality of the resulting combined hierarchy using the same stralcgy applicd in section 3. It is interesting to note that in the merged hicrarchy, all the absent hypernym problems (including absence due to or-heads) have been eliminated, since in every case at least one of the five dictionaries gives a valid hypernym. In addition, almost all of the attachments too high in the hicrarchy and missing overlaps have disappeared, although a few cases remain $(5 \%$ and $1 \%$, respectively). None of the dictionaries, for instance, gives pot as the hypemym of teapot, although three of the five dictionaries give pot as the hypernym of coffeepot. A larger dictionary database would enable the elimination of many of these remaining imperfections (for cxample, New Penguin English Dictionary, not included in our database, gives pot as a hypernym of leapot).

Merging dictionaries on a large scale assumes that it is possible to automatically map senses across them. For our small sample, we mapped senses among dictionaries by hand. We describe elsewhere a promising method to automatically accomplish sense mapping, using a spreading activation algorithm (Ide and Veronis, 1990).

\subsection{Covert categories}

There remain a number of circularly-defined hypernyms in the combined taxonomy, which demand additional consideration on theoretical grounds. Circularly-defined terms tend to appear when lexicographers lack terms to designate certain concepts. The fact that "it is not impossible for what is intuitively recognized as a conceptual catcgory to be without a label" has already been noted (Cruse, 1986, p. 147). The lack of a specific term for a recognizable concept tends to occur more frequently at the higher levels of the hierarchy (and at the very lowest and most specific levels as well--c.g., there is no tcrm to designate forks with two prongs). This is probably because any language includes the most terms at the generic level (Brown, 1958), that is, the level of cveryday, ordinary terms for objects and living things (dog, pencil, house, etc.).

Circularity, as wcll as the use of or-conjoincd terms at the high levels of the hicrarchy, results largely from the lexicographers' efforts to approximate the terms they lack. For example, there is no clear term to denote that catcgory of objects which fall under any of the terms utensil, tool, implement, instrument, although this concept secms to exist. Clearly, these terms are not strictly synonymous--there are, for cxample, utensils that onc would not call tools (e.g., a colander). If a term, let us say X, for the concept existed, then the definitions for utensil, tool, implement, and instrument 
could simply read "an X that...". Since this is not the casc, lexicographers define each term with a list including the others, which enables the delincation of a concept which encompasses all of them.

One way to resolve difficulties at the higher levels of extracted hierarchies is to introduce "coven categories", that is, concepts which do not correspond to any particular word. We therefore do not merge circular terms into a single concept, but instead creatc a common "covert" hypernym for all of them. In this way, tool, utensil, implement; and instrument each appear in the hierarchy as kinds of INSTRUMENTALOBJECT (covert categories names are capitalized).

We need a means to determine when and where covert catcgories are necessary. Circularities in dictionary definitions clearly indicate the presence of covert catcgories. However, we obviously cannot use a single dictionary to determine them, because the loops contained in one dictionary rarcly include all of the terms that may be involved in the "constellation" representing a given covert category. For instance, the CED contains the loop tool-implement-utensil, while the COBUILD contains a loop for tool-instrument; this provides strong evidence that all four terms should be involved in a constellation. Supporting information can be derived by looking at the hyponyms for each of the candidate terms in different dictionaries. The word fork, for example, is defined as tool (COBUILD), implement (CED, OALD, W9), and instrument ( $L D O C E)$, while spoon is defined as object (COBUILD), utensil (CED, OALD), lool (LDOCE) and implement (W9), which adds further support to the idea that tool, utensil, instrument, and implement belong to the same constellation.

Even if it is relatively easy to automatically detect circularities, the final determination of which covert categories to create and the terms that are involved in them must be done manually. However, this task is not as daunting as it may first appear, since it involves only the higher levels of the hicrarchy, and likely involves a relatively small number of covert categories.

\subsection{Summary}

By merging five dictionaries, all but $6 \%$ of the problems found in individual dictionaries were climinatcd (figure 8). This result is made clear in figure 10, which includes the same small subset of the sample corpus as in the individual hierarchies given in figure 7. Although there remain a few imperfections, the combined hierarchy is much more accurate and complete, and therefore more useful, than the hierarchy derived from any one of the dictionaries alone.

\section{CONCLUSION}

The results of our study show that dictionaries can be a reliable source of automatically extracted semantic information. Merging information from scveral dictionaries improved the quality of extracted information to an acceptable level. However, these results were obtained for a selected corpus representing a best case situation. It is likely that different results will be obtained for larger, less restricted cases. Our results suggest that this is an encouraging line of research to pursue for refining automatically extracted information.

\section{REFERENCES}

AKKERMAN, E., MASEREeUW, P. C., MeiJs, W. J. (1985). Designing a computcrized lexicon for linguistic purposes. ASCOT Report No. I, Rodopi, Amstcrdam.

AMSLER, R. A. (1980). The struciure of the Merriam-Websier Pocket Dictionary. Ph. D. Diss., U. Texas at Austin.

B ROWN, R. W. (1958) How shall a thing be called? Psychological Review, 65, 14-21.

BYRD, R. J. (1989) Discovering relationships among word senses. Proc. Sth Conf. UW Centre for the New OED, Oxford. $67-79$.

BYRd, R. J., CAlzolari, N., Ciodorow, M. S., Klavans, J. L., NEFF, M. S., RIZK, O. (1987) Tools and methods for computational linguistics. Computational Linguistics, 13. 3/4, 219-240.

CALZOLARI, N.(1984). Detecting pattems in a lexical data base. COLING'84, 170:173.

CHODOROW, M. S., BYRD. R. J., IIEIDORN, G. L. (1985). Extracting scmantic hicrarchics from a large on-line dictionary. Proc. 23rd Annual Conf. of the $A C L$, Chicago, 299-304.

CRUSE, D. A. (1986). Lexical semantics, Cambridge University Press, Cambridge.

IDE, N., M., VERONIS, J. (1990). Mapping Diclionaries: A Sprcading Activation Approach, Proc. 6th Conf. UW Centre for the New $O E D$. Waterloo, 52-64.

KlavaNS, J., Cliodorow, M., WaCIIOLdiR, N (1990). From dictionary to knowledge base via taxonomy. Proc. 6th Conf. UW Centre for the New OED, Watcrlow, 110-132.

LYONS, J. (1963) Siructural semantics. Blackwell, Oxford.

M ARKOWITZ, J., AHLSWEDE, T., EVENS, M. (1986). Semantically significant patterns in dictionary definitions. Proc. 24rd Annual Conf. of the ACL, New York, 112-119.

NAKAMURA, J., NAGAO, M. (1988). Tixtraction of semantic information from an ordinary English dictionary and its cvaluation. COLNG'88, 459-464.

VERONIS, J., IDE, N., M. (1990). Word Sense Disambiguation with Very Large Neural Networks Extracted from Machine Readable Dictionarics. COLING'90, Ilelsinki.

WILKS, Y., D. FASS, C. GUO, J. MACDONALD, T. PLATE, B. SLATOR (1990). Providing Machine Tractable Dictionary Tools. Machine Translation,5, 99-154.

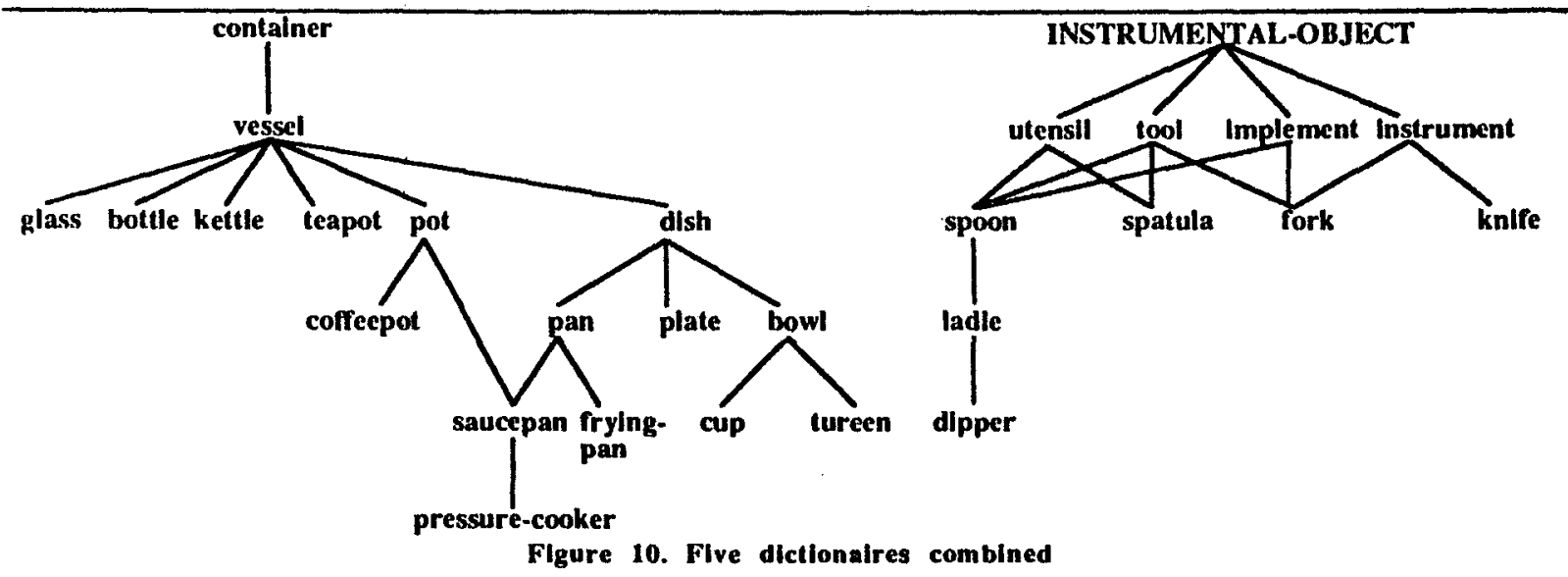

